\title{
SURGICAL TECHNIQUES
}

\section{Lateral Elbow Exposures: The Extensor Digitorum Communis Split Compared With the Kocher Approach}

Randa Berdusco, MD, FRCSC, Hakim Louati, MASc, William Desloges, MD, FRCSC, Steven R. Papp, MD, MSc, FRCSC, J Whitcomb Pollock, MD, MSc, FRCSC

Published outcomes of this procedure can be found at: $J$ Bone

Joint Surg Am. 2014 Mar 5;96

(5):387-93.

\section{Introduction}

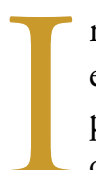

$\mathrm{n}$ comparison with the frequently used modified Kocher approach, the extensor digitorum communis (EDC) splitting approach allows improved access to the anterior half of the radial head ${ }^{1-3}$, which is most commonly fractured ${ }^{4}$, while reducing the risk of iatrogenic injury to the lateral collateral ligament ${ }^{1}$.

The Kocher approach was originally described as a posterolateral capsulotomy in line with the anconeus, posterior to the central portion of the lateral ulnar collateral ligament ${ }^{5}$. The modified Kocher approach is now commonly used for radial head fracture fixation, performed between the anconeus and extensor carpi ulnaris muscular interval ${ }^{1,6-10}$. The extensor carpi ulnaris muscle is then mobilized anteriorly, followed by a capsulotomy along the anterior border of the lateral ulnar collateral ligament ${ }^{7-9}$. Anterior exposure of the radial head can be limited through this approach ${ }^{1-3}$, resulting in poor visibility and difficulty with screw placement, especially in muscular patients. If further exposure is required, the common extensor origin (extensor carpi ulnaris, EDC, and extensor carpi radialis brevis tendons) can be detached from the lateral epicondyle, followed by detachment of the extensor carpi radialis longus and the brachioradialis from the supracondylar ridge as required ${ }^{11}$.

The EDC split is an alternative safe technique ${ }^{1-3}$ that provides greater exposure of the anterior half of the radial head for easier fixation of radial head fractures. This approach is lateral to the Kaplan interval, described between the extensor carpi radialis brevis and longus tendons and the $\mathrm{EDC}^{12}$. The EDC tendon is identified and bisected longitudinally, starting proximally at the lateral epicondylar origin and extending $25 \mathrm{~mm}$ distally from the radiocapitellar joint. The annular ligament and joint capsule are then incised collinear with the EDC split anterior to the equator of the capitellum to avoid

Disclosure: None of the authors received payments or services, either directly or indirectly (i.e., via his or her institution), from a third party in support of any aspect of this work. None of the authors, or their institution (s), have had any financial relationship, in the thirty-six months prior to submission of this work, with any entity in the biomedical arena that could be perceived to influence or have the potential to influence what is written in this work. Also, no author has had any other relationships, or has engaged in any other activities, that could be perceived to influence or have the potential to influence what is written in this work. The complete Disclosures of Potential Conflicts of Interest submitted by authors are always provided with the online version of the article. 
injury to the lateral collateral ligament posteriorly ${ }^{6,7}$ and resultant posterolateral rotary instability ${ }^{13,14}$. If greater exposure is needed, the anterior half of the EDC tendon and the extensor carpi radialis brevis tendon are detached proximally from the lateral epicondyle followed by detachment of the extensor carpi radialis longus and the brachioradialis origins from the supracondylar ridge ${ }^{1,3}$.

Both the modified Kocher approach and the EDC splitting approach can be performed through an oblique lateral incision overlying the radiocapitellar joint or a posterior longitudinal incision with a raised full-thickness lateral fasciocutaneous flap depending on the presence of concomitant injuries or surgeon preference ${ }^{1,3}$. Comparisons of the modified Kocher and EDC splitting approaches are demonstrated in Figures 1-A and 1-B, followed by a more detailed description and video illustration of both surgical techniques.

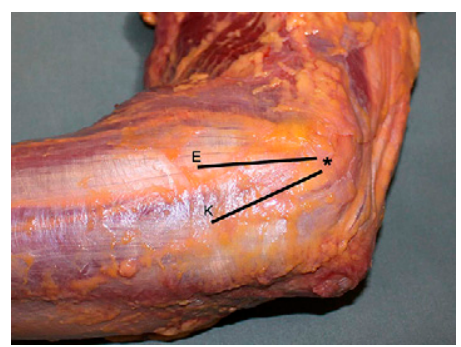

Fig. 1-A

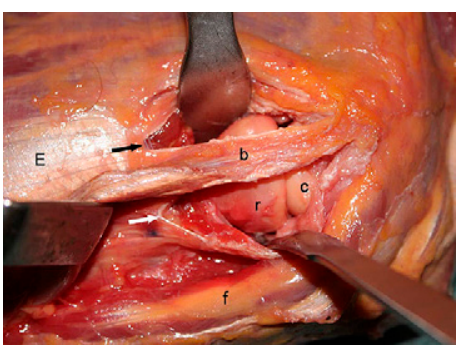

Fig. 1-B

Figs. 1-A and 1-B Lateral view of a left elbow specimen showing the EDC splitting approach and the Kocher approach. (Reproduced from: Desloges W, Louati H. Papp SR, Pollock JW. Objective analysis of lateral elbow exposure with the extensor digitorum communis split compared with the Kocher interval. J Bone Joint Surg Am. 2014 Mar 5; 96[5]:387-93.) Fig. 1-A The EDC splitting approach was performed by bisecting the EDC tendon longitudinally (line E) starting at its origin on the lateral epicondyle ${ }^{*}$ ). The Kocher approach was performed through the interval between the anconeus and extensor carpi ulnaris (line K). Fig. 1-B The EDC splitting approach (black arrow) and Kocher approach (white arrow) performed concurrently on the cadaveric specimen. The bridge of tissue (b) is made up of the inferior half of the EDC and the extensor carpi ulnaris. $E=E D C, r=$ radial head, $c=$ capitellum, and $f=f a t$ stripe between the anconeus and extensor carpi ulnaris. Note that retraction is not optimized for either approach. 


\section{Step 1: Make the Incision (Modified Kocher Approach)}

\section{Make an oblique 7-cm lateral incision beginning at the proximal edge of the lateral epicondyle and} extending distally over the center of the radial head toward the posterior ulnar border of the extensor carpi ulnaris muscle belly (Video 1).

- With the patient under general or regional anesthesia, examine the injured elbow and assess for ligamentous stability.

- Position the patient supine with the arm slightly abducted on a radiolucent arm board or hand table. Alternatively, the arm may be placed over the patient's chest with a bump under the ipsilateral scapula.

- After limb exsanguination, inflate a tourniquet.

- Pronate the forearm and flex the elbow to $90^{\circ}$.

- Identify landmarks: the lateral supracondylar ridge, lateral epicondyle, radial head, and radiocapitellar joint. Lateral thumb pressure with supination and pronation of the forearm aids in the identification of the radial head and radiocapitellar joint ${ }^{15}$.

- Make an oblique 7-cm lateral incision beginning just proximal to the center of the lateral epicondyle and extending distally over the center of the radial head toward the posterior ulnar border of the extensor carpi ulnaris muscle belly (Figs. 2-A and 2-B).

- If an extensile approach is required for access to the medial and lateral aspects of the elbow, a posterior incision with a raised full-thickness lateral fasciocutaneous flap may be utilized. In this scenario, place the patient supine with the arm over the chest.

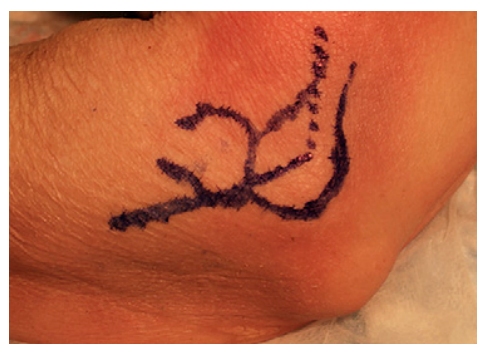

Fig. 2-A

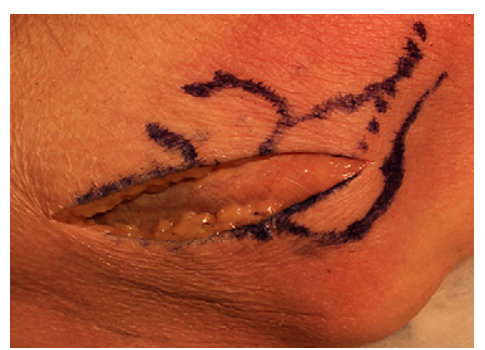

Fig. 2-B

Video 1 Lateral view of a left cadaveric elbow specimen depicting the modified Kocher surgical incision and development of full-thickness fasciocutaneous flaps.

Figs. 2-A and 2-B Photographs of a cadaveric left elbow specimen illustrating the modified Kocher approach. Fig. 2-A The marked lateral oblique incision overlies the outlined radiocapitellar joint. The dotted line represents proximal extension if required. Fig. 2-B Lateral oblique incision. 


\section{Step 2: Develop the Interval Between the Anconeus and the Extensor Carpi Ulnaris}

\section{Identify and develop the intermuscular interval between the anconeus and the extensor carpi ulnaris} (Videos 2 and 3).

- Identify the interval between the anconeus (radial nerve) and the extensor carpi ulnaris (posterior interosseous nerve). It is easier to identify from a distal to proximal direction as the muscular fibers blend together proximally.

- Locate the characteristic thin fat stripe that is often within this intermuscular interval to further aid with identification (Fig. 3-A).

- Divide the intermuscular deep fascia between the anconeus and the extensor carpi ulnaris, beginning at the lateral epicondyle and extend distally (Fig. 3-B).

- Retract the anconeus posteriorly.

- Mobilize and then retract the extensor carpi ulnaris anteriorly off the joint capsule.

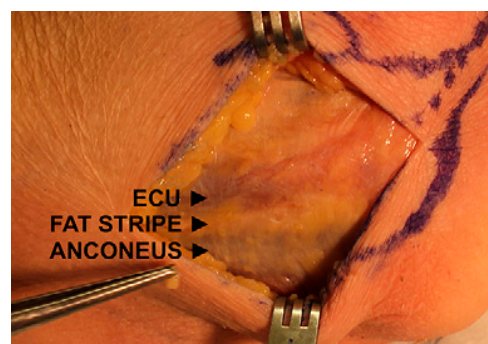

Fig. 3-A

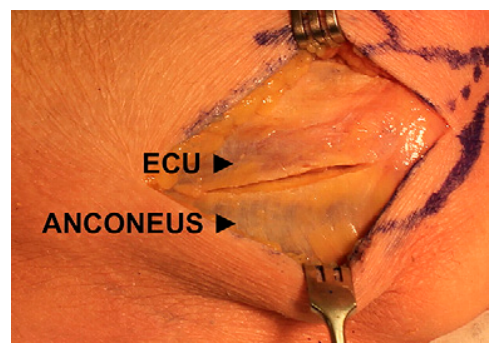

Fig. 3-B

Video 2 Lateral view of a left cadaveric elbow specimen showing the identification of the lateral elbow musculature and characteristic fat stripe. Video 3 Lateral view of a left cadaveric elbow specimen. The interval between anconeus posteriorly and extensor carpi ulnaris anteriorly in the modified Kocher approach is developed.

Figs. 3-A and 3-B Localizing and developing the interval in the modified Kocher approach. Fig. 3-A The characteristic thin fat stripe is used as a divisional landmark, and the interval between the extensor carpi ulnaris (ECU) and the anconeus is localized. Fig. 3-B The interval between the extensor carpi ulnaris (ECU) and the anconeus is developed. 


\section{Step 3: Perform the Lateral Elbow Capsulotomy}

\section{Longitudinally incise the lateral elbow capsule and annular ligament anterior to the lateral ulnar} collateral ligament (Video 4).

- Ensure that the forearm is fully pronated to protect the posterior interosseous nerve.

- Longitudinally incise the lateral elbow capsule and annular ligament anterior to the lateral ulnar collateral ligament (Fig. 4-A). The lateral ulnar collateral ligament can be found in line with and deep to the anterior fibers of the anconeus. Begin at the lateral epicondyle and extend over the radial head equator.

- Place two small Hohmann retractors within the radiocapitellar joint to gently displace soft tissues anteriorly and posteriorly (Fig. 4-B).

- Do not extend the capsular incision beyond the distal edge of the annular ligament or retract aggressively, to avoid injury to the posterior interosseous nerve.

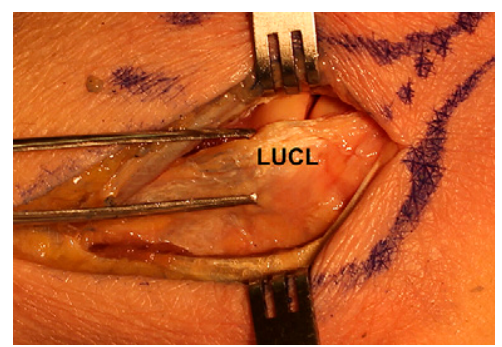

Fig. 4-A

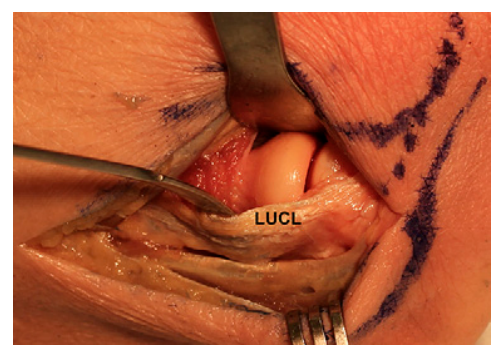

Fig. 4-B

Video 4 Lateral view of a left cadaveric elbow specimen illustrating the lateral elbow capsulotomy anterior to the lateral ulnar collateral ligament in the modified Kocher approach.

Figs. 4-A and 4-B Capsulotomy in the modified Kocher approach. Fig. 4-A The lateral elbow capsulotomy is performed anterior to the lateral ulnar collateral ligament (LUCL) (defined by forceps), exposing the radiocapitellar joint. Fig. 4-B Two small Hohmann retractors are utilized for ideal capsular retraction, further exposing the radiocapitellar joint. 


\section{Step 4: The Extended Modified Kocher Approach}

Extend the exposure by elevating the common extensor origin (extensor carpi radialis brevis, EDC, and extensor carpi ulnaris) proximally off the lateral epicondyle and reflect it anteriorly (Video 5).

- Extend the lateral skin incision proximally in line with the lateral supracondylar ridge as required (Fig. 5-A).

- Identify and protect the origin of the lateral collateral ligament on the lateral epicondyle.

- Sharply dissect the common extensor origin (extensor carpi radialis brevis, EDC, and extensor carpi ulnaris) off the lateral epicondyle and reflect it anteriorly (Figs. 5-B and 5-C).

- For further exposure, dissect the extensor carpi radialis longus and brachioradialis origins from the supracondylar ridge and reflect them anteriorly.

- Leave $5 \mathrm{~mm}$ of fascia attached to the humerus to facilitate repair and closure.

- Extend the previous capsulotomy proximally along the anterior border of the lateral supracondylar ridge.

- With the elbow flexed to minimize tension, place intracapsular retractors deep to the joint capsule anteriorly.

- Release the superior capsular attachment off the anterior aspect of the humerus, as required.

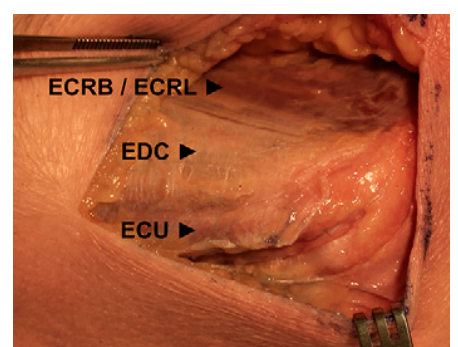

Fig. 5-A

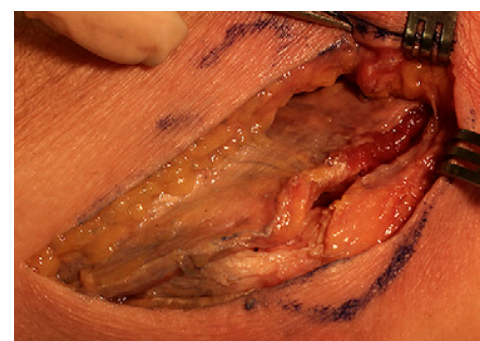

Fig. 5-B

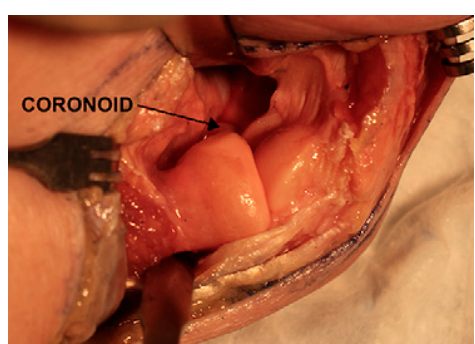

Fig. $5-\mathrm{C}$

Video 5 Lateral view of a left cadaveric elbow specimen illustrating the extended modified Kocher approach.

Figs. 5-A through 5-C Extended modified Kocher approach. Fig. 5-A The incision is extended proximally. ECRB = extensor carpi radialis brevis, ECRL $=$ extensor carpi radialis longus, and ECU = extensor carpi ulnaris. Fig. 5-B The common extensor origin is elevated proximally off the lateral epicondyle. Fig. 5-C The common extensor origin is reflected anteriorly. For increased exposure, intracapsular retractors are carefully placed anteriorly and the superior capsular attachment is released from the anterior aspect of the humerus as required. 


\section{Step 5: Make the Incision (EDC Splitting Approach)}

Make a longitudinal oblique 5 to 6-cm lateral incision beginning at the proximal edge of the lateral epicondyle and extending distally over the radial head toward the Lister tubercle (Video 6).

- Refer to Step 1 for patient positioning and identification of landmarks.

- Make a longitudinal oblique 5 to $6-\mathrm{cm}$ lateral incision beginning just proximal to the center of the lateral epicondyle and extending distally over the radial head toward the Lister tubercle ${ }^{16}$ (Figs. 6-A and 6-B).

- Alternatively, a posterior incision with a raised full-thickness lateral fasciocutaneous flap may also be utilized.

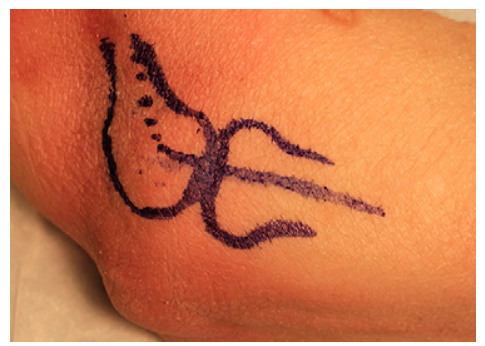

Fig. 6-A

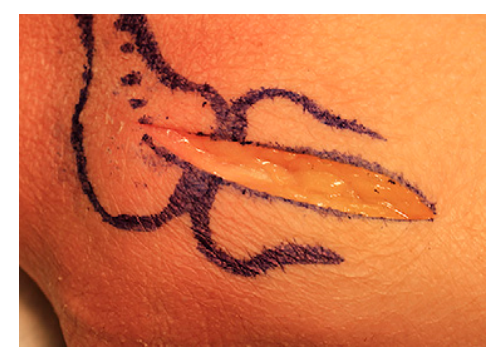

Fig. 6-B

Video 6 Lateral view of a right cadaveric elbow specimen depicting the EDC split surgical incision.

Figs. 6-A and 6-B Photographs of a cadaveric right elbow illustrating the EDC splitting approach. Fig. 6-A Marked lateral oblique incision overlying the outlined radiocapitellar joint. Dotted line represents proximal extension if required. Fig. 6-B The lateral oblique incision. 


\section{Step 6: Identify and Split the EDC}

\section{The EDC tendon is identified and bisected longitudinally starting proximally at its origin on the lateral} epicondyle and extending $20 \mathrm{~mm}$ distally from the radiocapitellar joint (Videos 7 and 8).

- Identify the EDC tendon (posterior interosseous nerve), as it runs in line from the lateral epicondyle toward the Lister tubercle ${ }^{16}$. We find that the EDC has a characteristic appearance represented by a relatively white tendinous structure separated with fine longitudinal red muscular striations, whereas the extensor carpi ulnaris and the extensor carpi radialis brevis tendons have a more homogeneous red muscular appearance with few striations (Figs. 5-A, 7-A, and 7-B).

- With the forearm fully pronated, longitudinally bisect the EDC tendon centered over the radial head. Start at its origin on the lateral epicondyle and extend $20 \mathrm{~mm}$ distally from the radiocapitellar joint (Fig. 8).

- Retract the split EDC tendon anteriorly and posteriorly.

- Bluntly develop the plane overlying the anterolateral joint capsule.

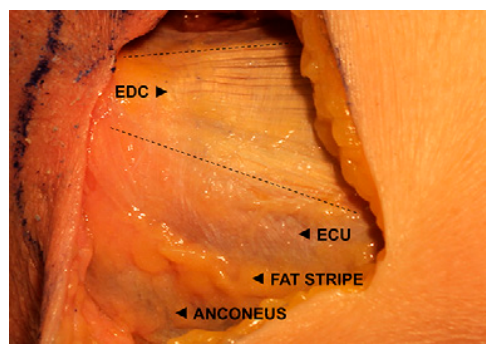

Fig. 7-A

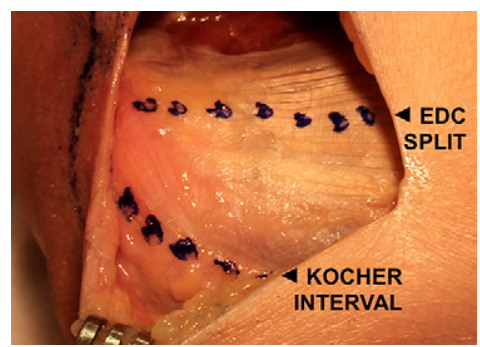

Fig. 7-B

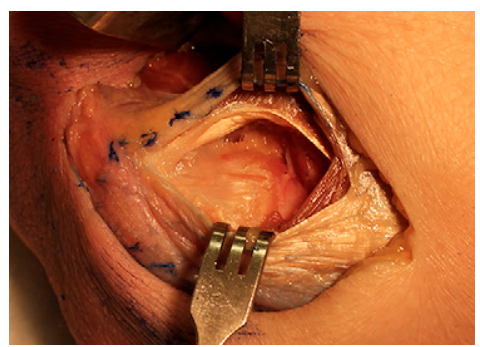

Fig. 8

Video 7 Lateral view of a right cadaveric elbow specimen showing the identification of the lateral elbow musculature (emphasizing identification of EDC) and characteristic fat stripe.

Video 8 Lateral view of a right cadaveric elbow specimen. The longitudinal bisection of the EDC tendon and the collinear incision of the annular ligament and joint capsule of the EDC split approach are illustrated.

Figs. 7-A and 7-B Identification of EDC tendon in the EDC splitting approach and comparison with the modified Kocher approach. Fig. 7-A The EDC tendon is identified by its characteristic white tendinous appearance (with fine red muscular striations). The EDC borders are represented by dotted lines. ECU = extensor carpi ulnaris. Fig. 7-B A comparison of the EDC splitting approach (superior dotted line bisecting the EDC tendon) and the modified Kocher approach (inferior dotted line representing the interval between the extensor carpi ulnaris and the anconeus).

Fig. 8 In the EDC splitting approach, a bisecting longitudinal split of the EDC tendon, exposing the lateral radiocapitellar joint capsule, is performed. The collinear capsular indentation represents the location of the capsulotomy depicted in Figure 9-A. 


\section{Step 7: Perform the Lateral Elbow Capsulotomy}

The annular ligament and joint capsule are then incised collinear with the EDC split anterior to the equator of the capitellum (Video 8).

- Ensure that the forearm is fully pronated to protect the posterior interosseous nerve.

- Incise the annular and radial collateral ligaments and joint capsule in a collinear fashion with the EDC split. Begin proximally $2 \mathrm{~mm}$ anterior to the equator of the capitellum to avoid iatrogenic injury to the origin of the lateral ulnar collateral ligament (Fig. 9-A).

- Place two small Hohmann retractors within the radiocapitellar joint to gently displace soft tissues anteriorly and posteriorly (Fig. 9-B).

- Do not extend the capsular incision beyond the distal edge of the annular ligament or retract aggressively, to avoid injury to the posterior interosseous nerve.

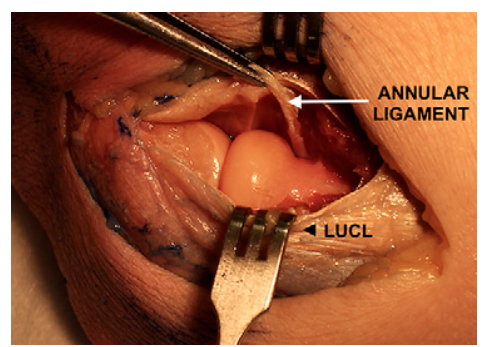

Fig. 9-A

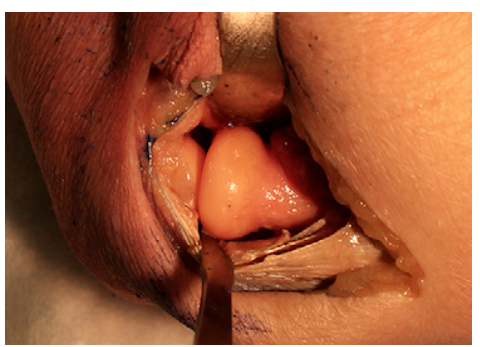

Fig. 9-B

Figs. 9-A and 9-B Capsulotomy in the EDC splitting approach. Fig. 9-A The lateral elbow capsulotomy is performed anterior to the equator of the capitellum in line with the EDC split exposing the radiocapitellar joint. LUCL = lateral ulnar collateral ligament. Fig. 9-B An anteriorly placed capsular retractor demonstrates the increased exposure to the anterior radial head. Compare with the modified Kocher exposure of the radial head in Figure 4-B. 


\section{Step 8: Extended EDC Splitting Approach}

\section{Extend the exposure by detaching the anterior half of the EDC tendon and the extensor carpi radialis brevis tendon from the lateral epicondyle (Video 9).}

- Extend the lateral skin incision proximally in line with the lateral supracondylar ridge as required.

- Sharply dissect the anterior half of the EDC tendon as well as the extensor carpi radialis brevis and longus tendons off the lateral epicondyle and reflect them anteriorly (Figs. 10-A and 10-B).

- For further exposure, dissect the extensor carpi radialis longus and brachioradialis origins from the supracondylar ridge and reflect them anteriorly.

- Leave $5 \mathrm{~mm}$ of fascia attached to the humerus to facilitate repair and closure.

- Extend the previous capsulotomy proximally along the anterior border of the lateral supracondylar ridge.

- With the elbow flexed to minimize tension, place intracapsular retractors deep to the joint capsule anteriorly (Fig. 10-C).

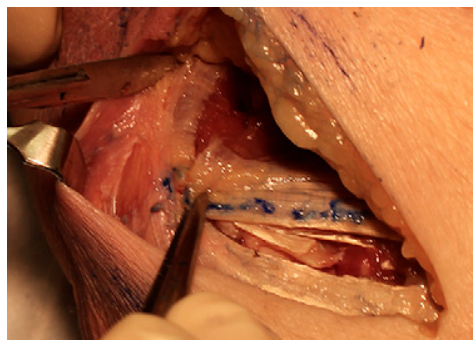

Fig. 10-A

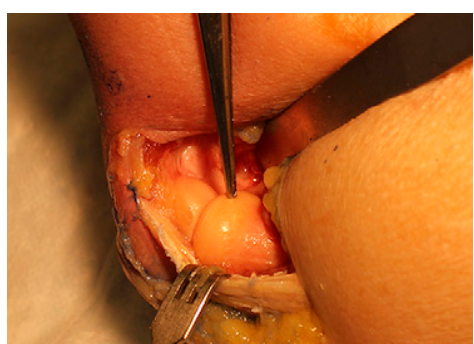

Fig. 10-D

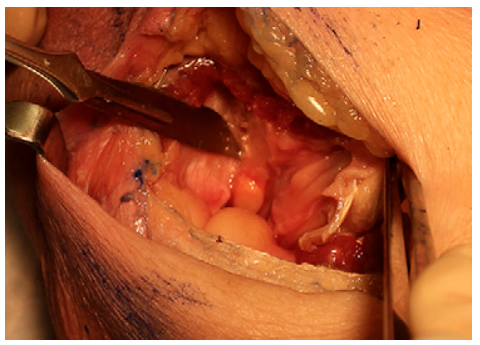

Fig. 10-B

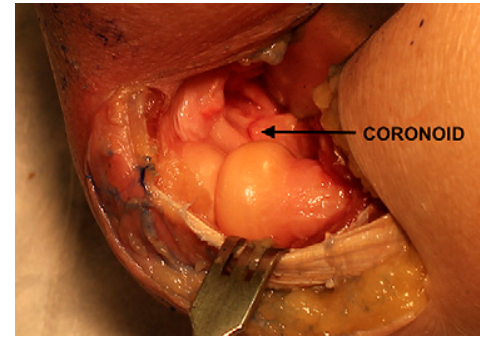

Fig. $10-\mathrm{C}$

Video 9 Lateral view of a right cadaveric elbow specimen illustrating the extended EDC splitting approach.

Figs. 10-A through 10-D The extended EDC splitting approach. Fig. 10-A The lateral skin incision is extended proximally in line with the lateral supracondylar ridge. The anterior half of the EDC tendon and the common extensor origin are dissected off the lateral epicondyle. Fig. 10-B The anterior half of the EDC tendon and the common extensor origin are retracted anteriorly. Fig. 10-C The anterior half of the EDC tendon as well as the extensor carpi radialis brevis and longus tendons are reflected anteriorly utilizing a carefully placed retractor for increased exposure. Compare with the extended modified Kocher exposure in Figure 5-C. Fig. 10-D Forceps demonstrate the excellent anterior to posterior access of the radial head for screw placement (perpendicular to the fracture line) frequently required for anterolateral fracture fixation. 


\section{Step 9: Layered Closure}

\section{Perform an interrupted layered closure.}

- Anatomic closure of both the modified Kocher and the EDC splitting approach is performed in an interrupted, layered fashion from deep to superficial, beginning with the lateral joint capsule.

\section{Results}

In our recent cadaveric study, we quantitatively compared the modified Kocher and EDC splitting approaches in order to determine which provided the greatest exposure of the anterior aspect of the radial head, which is most commonly fractured $^{17}$. The EDC splitting approach provided greater visualization of the anterior surface area of the radial head (median, 100\%) compared with the modified Kocher approach $(68 \%$; $<<0.05)$. The posterolateral and anterolateral quadrants of the radial head were visualized equally well between the two approaches; however, visualization of the anteromedial quadrant was significantly improved with the EDC splitting approach $(\mathrm{p}<0.05)$. Evaluation of the extended modified Kocher and extended EDC splitting approaches revealed that they provided comparable visualization of the distal end of the humerus, capitellum, radial head, and coronoid process.

Adequate exposure of the fractured radial head while minimizing soft-tissue disruption is crucial for fragment reduction, accurate placement of stable fixation, and overall successful surgical management. Our clinical experience supports the notion that the EDC splitting approach provides easier and greater access to isolated radial head fractures requiring fixation, as the majority of such fractures extend to the anteromedial quadrant ${ }^{4}$ (Fig. 10-D). We believe that, in comparison with a modified Kocher approach for radial head fracture fixation, there is less aggressive soft-tissue retraction and a lower likelihood that proximal extension of the exposure is required through an EDC split, especially in muscular patients.

In the case of an existing radial head deformity or prior acute fracture, no discrete adjustments to the EDC splitting approach are required. The surgeon may approximate the center of the radial head through repetitive forearm pronation and supination before performing the capsular incision in pronation approximately $2 \mathrm{~mm}$ anterior to the equator of the capitellum in order to avoid injury to the lateral ulnar collateral ligament.

\section{What to Watch For}

\section{Indications}

- Radial head fracture reduction and fixation.

- Radial head arthroplasty.

- Radial head or fragment excision.

- Capitellar fracture reduction and fixation.

- Anterior elbow debridement and capsular release.

\section{Contraindications}

- For the EDC splitting approach: Associated traumatic lateral ulnar collateral ligament and common extensor tendon injuries and/or disruption (typically avulsed from the lateral epicondyle). To optimize exposure, these injuries are better addressed using a Kocher approach and/or working through the zone of injury ${ }^{1,3,18}$.

\section{Pitfalls \& Challenges}

- Identification of the EDC tendon. It runs in line from the lateral epicondyle toward the Lister tubercle. The EDC has a characteristic appearance represented by a relatively white tendinous structure separated with fine longitudinal red muscular striations, whereas the extensor carpi ulnaris and extensor carpi radialis brevis tendons have a more muscular (red) appearance, with few striations (Fig. 5-A). 
- Disruption of the lateral ulnar collateral ligament insertion. It is important to palpate the borders of the radial head to ensure that the EDC incision is anterior to the equator of the radial head and that the lateral ulnar collateral ligament is protected posteriorly. Ensure that the incision is proximal to the equator of the capitellum (we suggest $2 \mathrm{~mm}$ ) to avoid iatrogenic injury to the lateral ulnar collateral ligament when performing the lateral capsulotomy.

- Proximity of the posterior interosseous nerve. Ensure that the forearm is maintained in pronation to move the nerve away from the surgical approach, both distally and medially ${ }^{19,20}$. Avoid excessive or aggressive softtissue retraction, most notably anteriorly. Do not extend the capsular incision beyond the distal aspect of the annular ligament.

\section{Clinical Comments}

- Incision selection. Both the modified Kocher and the EDC splitting approach can be performed through a lateral incision overlying the radiocapitellar joint or a posterior incision with a raised lateral fasciocutaneous flap depending on the presence of concomitant injuries or surgeon preference ${ }^{1,3}$. We base our selection on preoperative imaging to evaluate for additional fractures in combination with an intraoperative examination under anesthesia to assess elbow stability. If there is no anteromedial coronoid fracture and the medial collateral ligament is stable, a lateral incision is utilized. If medial exposure is required for the repair of an anteromedial coronoid fracture or a medial collateral ligament injury, then a posterior incision is used. Dual (medial and lateral) incisions are also acceptable. A posterior incision disrupts fewer cutaneous nerves, which potentially lowers the risk of postoperative paresthesias or painful neuroma formation ${ }^{21}$. A lateral incision has a theoretically lower risk for the development of wound complications as it is more direct, involving smaller fasciocutaneous flaps.

- For radial head fractures associated with traumatic lateral ulnar collateral ligament or common extensor ruptures, we recommend using the modified Kocher approach. This allows the surgeon to work through the zone of injury, which characteristically involves an avulsion of the origin of the lateral ulnar collateral ligament from the lateral epicondyle in order to access the radial head fracture and facilitate lateral ligamentous repair ${ }^{1,3,18}$.

- Protection of the posterior interosseous nerve. In a cadaveric series, the shortest measured distance from the radiocapitellar joint to the posterior interosseous nerve during the use of an EDC splitting approach with the forearm pronated was $29 \mathrm{~mm}$, with an average safe zone (and standard deviation) of $48.2 \pm 7.9 \mathrm{~mm}$ from the radiocapitellar joint ${ }^{22}$. To ensure protection of the posterior interosseous nerve, we limit the distal extension of the EDC split to $20 \mathrm{~mm}$ from the radiocapitellar joint. In our experience, this routinely provides adequate exposure. Proximal splitting of the tendon minimizes potential denervation as the EDC is innervated in a retrograde fashion more distally by the recurrent branch of the EDC muscle (also known as the first branch of the posterior interosseous nerve $)^{23}$.

- The proximal extended approaches may be required for additional exposure in muscular patients or for the reduction and fixation of coronal coronoid fractures.

- The Kaplan approach is just medial to the EDC splitting approach, between the extensor carpi radialis brevis and longus tendons and the EDC. It also provides anterior access to the radial head while avoiding iatrogenic injury to the lateral ulnar collateral ligament. Kaplan similarly advised maintaining the forearm in pronation and not extending the incision distally beyond 2 in $(5 \mathrm{~cm})$ from the articular surface of the radial head in order to avoid injury to the posterior interosseous nerve ${ }^{12}$.

\section{Acknowledgment}

NoтE: Video demonstrations and narration by J. Whitcomb Pollock. 
Randa Berdusco, MD, FRCSC

Hakim Louati, MASc

William Desloges, MD, FRCSC

Steven R. Papp, MD, MSc, FRCSC

J Whitcomb Pollock, MD, MSc, FRCSC

The Ottawa Hospital, University of Ottawa, 501 Smyth Road, Ottawa, ON K1H 8L6, Canada

\section{References}

1. Rosenblatt Y, Athwal GS, Faber KJ. Current recommendations for the treatment of radial head fractures. Orthop Clin North Am. 2008 Apr;39(2):173-85, vi. 2. Redler LH, Hsu SH, Levine WN. Management of radial head fractures. In: Levine WN, Ahmad CS, Cadet ER, editors. Shoulder and elbow trauma. London: JP Medical Ltd; 2012. p 179-88.

3. King GJW. Fractures of the head of the radius. In: Wolfe SW, Hotchkiss RN, Pederson WC, Kozen SH, editors. Green's operative hand surgery, 6th ed. Philadelphia: Churchill Livingstone Elsevier; 2011. p 783-819.

4. van Leeuwen DH, Guitton TG, Lambers K, Ring D. Quantitative measurement of radial head fracture location. J Shoulder Elbow Surg. 2012 Aug;21(8): 1013-7. Epub 2011 Nov 9.

5. Kocher T. Text-book of operative surgery. New York: Macmillan; 1911. p 313.

6. Hotchkiss RN. Displaced fractures of the radial head: internal fixation or excision? J Am Acad Orthop Surg. 1997 Jan;5(1):1-10.

7. Patterson SD, Bain Gl, Mehta JA. Surgical approaches to the elbow. Clin Orthop Relat Res. 2000 Jan;370:19-33.

8. Morrey BF. Surgical exposures of the elbow. In: Morrey BF, Sanchez-Sotelo J, editors. The elbow and its disorders, 4th ed. Philadelphia: Saunders Elsevier; 2009. p 115-22.

9. Morrey BF. Master techniques in orthopaedic surgery: the elbow, 2nd ed. Philadelphia: Lippincott Williams \& Wilkins; 2002. p 83-102.

10. Cheung EV, Steinmann SP. Surgical approaches to the elbow. J Am Acad Orthop Surg. 2009 May;17(5):325-33.

11. McKee MD, Jupiter JB, Bamberger HB. Coronal shear fractures of the distal end of the humerus. J Bone Joint Surg Am. 1996 Jan;78(1):49-54. 12. Kaplan EB. Surgical approach to the proximal end of the radius and its use in fractures of the head and neck of the radius. J Bone Joint Surg Am. 1941 Jan;23(1):86-92.

13. Dunning CE, Zarzour ZD, Patterson SD, Johnson JA, King GJ. Muscle forces and pronation stabilize the lateral ligament deficient elbow. Clin Orthop Relat Res. 2001 Jul;388:118-24.

14. Hall JA, McKee MD. Posterolateral rotatory instability of the elbow following radial head resection. J Bone Joint Surg Am. 2005 Jul;87(7):1571-9. 15. Hoppenfeld S, deBoer P, Buckley R, editors. Surgical exposures in orthopaedics: the anatomic approach, 4th ed. Philadelphia: Lippincott Williams \& Wilkins; 2009. p 133-45.

16. Rosenblatt Y, King GJ. Radial head replacement. In: Weisel SW, editor. Operative techniques in orthopaedic surgery. Philadelphia: Lippincott Williams \& Wilkins; 2011. p 3353-4.

17. Desloges W, Louati H, Papp SR, Pollock JW. Objective analysis of lateral elbow exposure with the extensor digitorum communis split compared with the Kocher interval. J Bone Joint Surg Am. 2014 Mar 5;96(5):387-93.

18. McKee MD, Pugh DM, Wild LM, Schemitsch EH, King GJ. Standard surgical protocol to treat elbow dislocations with radial head and coronoid fractures. Surgical technique. J Bone Joint Surg Am. 2005 Mar;87(Pt 1)(Suppl 1):22-32.

19. Strachan JC, Ellis BW. Vulnerability of the posterior interosseous nerve during radial head resection. J Bone Joint Surg Br. 1971 May;53(2):320-3. 20. Diliberti T, Botte MJ, Abrams RA. Anatomical considerations regarding the posterior interosseous nerve during posterolateral approaches to the proximal part of the radius. J Bone Joint Surg Am. 2000 Jun;82(6):809-13.

21. Dowdy PA, Bain Gl, King GJ, Patterson SD. The midline posterior elbow incision. An anatomical appraisal. J Bone Joint Surg Br. 1995 Sep;77(5):696-9. 22. Schimizzi A, MacLennan A, Meier KM, Chia B, Catalano LW 3rd, Glickel SZ. Defining a safe zone of dissection during the extensor digitorum communis splitting approach to the proximal radius and forearm: an anatomic study. J Hand Surg Am. 2009 Sep;34(7):1252-5.

23. Elgafy H, Ebraheim NA, Rezcallah AT, Yeasting RA. Posterior interosseous nerve terminal branches. Clin Orthop Relat Res. 2000 Jul;376:242-51. 